\title{
Measuring Temporal Preparation
}

\author{
Mariagrazia Capizzi and Ángel Correa
}

\section{1 \\ Introduction}

Temporal preparation is a fundamental cognitive ability that enables us to anticipate the moment in time (in the millisecond to second range) at which a relevant event might occur. At an operational level, this means that when stimulus onsets can be predicted, both accuracy and response time to such stimuli will be improved compared to stimuli with unpredictable onset (Correa, 2010; Nobre, Correa, \& Coull, 2007). Theoretically, temporal preparation is linked to the field of time perception since the ability to anticipate when something is going to occur does involve the capacity to compute the passage of time. It should be noted, however, that experiments on temporal preparation require using time rather than consciously estimating it (i.e., time estimation and reproduction tasks; e.g., Grondin, 2010; Ivry \& Hazeltine, 1995; Chapters 2 and 4, this volume).

Early studies of temporal preparation focused on the behavioral effects that the manipulation of the "foreperiod" (timing during which a warning signal precedes a target stimulus) had on participants' performance (Woodrow, 1914). Subsequent studies investigated whether temporal preparation could be voluntarily acquired following a symbolic and predictive temporal cue (e.g., Coull \& Nobre, 1998; Miniussi, Wilding, Coull, \& Nobre, 1999; Nobre, 2001). More recent work has explored temporal preparation driven by the presentation of regular rhythmic patterns (e.g., Cutanda, Correa, \& Sanabria, 2015; Jones, Moynihan, MacKenzie, \& Puente, 2002; Lange, 2010; Sanabria, Capizzi, \& Correa, 2011).

These complementary research approaches have identified the basic features of the temporal preparation effects that will be covered in the following sections of this chapter. Each section provides a brief description of one of the paradigms used to investigate temporal preparation along with an example of how to measure and interpret the behavioral data usually obtained in the studies employing such a paradigm. For a thorough review of the cognitive processes and neural mechanisms underlying temporal preparation, the reader is referred to Nobre and Coull (2010). 
In the foreperiod paradigm, a warning signal precedes a target stimulus, to which the participant is to respond (see Coull, 2009; Niemi \& Näätänen, 1981, for reviews). The foreperiod is the time interval between warning signal and target. It represents the period in which temporal preparation has been shown to take place. Consider, for example, the simplest case with only two possible foreperiods (e.g., $1000 \mathrm{~ms}$ - short vs. $3000 \mathrm{~ms}-\mathrm{long}$ ). The variability with which short and long foreperiods are presented in different trials will selectively influence participants' reaction times (RTs). When there is no variability and just one foreperiod is administered in a block of trials (i.e., fixed foreperiod design), it is typical to find shorter RTs with short foreperiods, a phenomenon known as the "fixed foreperiod effect" (e.g., Bausenhart, Rolke, \& Ulrich, 2008; Mattes \& Ulrich, 1997; Vallesi, McIntosh, Shallice, \& Stuss, 2009a). Conversely, when short and long foreperiods are randomly intermixed with equal probability within a block (i.e., variable foreperiod design), shorter RTs will be associated with long foreperiods (i.e., the "variable foreperiod effect"; hereafter simply referred to as the foreperiod effect; e.g., Drazin, 1961; Mento, Tarantino, Vallesi, \& Bisiacchi, 2015; Niemi \& Näätänen, 1981; Woodrow, 1914). The discrepant findings between fixed and variable foreperiod designs can be accounted for by two separate factors: time estimation and conditional probability of target occurrence.

On the one hand, in a fixed foreperiod paradigm, it is likely that participants use the warning signal as a reference to estimate the time at which the target stimulus will be presented (Klemmer, 1956, 1957; Niemi \& Näätänen, 1981). Given that uncertainty in time estimation increases as a function of the duration of the interval being estimated (Gibbon, 1977), it will be more difficult to anticipate the target onset in long than in short foreperiod blocks, which will lead to longer RTs in the long foreperiod blocks.

On the other hand, in a variable foreperiod paradigm, the conditional probability of target onset increases with the passage of time. Accordingly, participants learn that if the target does not appear at the short foreperiod, it will necessarily do so at the long foreperiod (Elithorn \& Lawrence, 1955). Under this "strategic" view of the foreperiod effect, participants' temporal expectation would be higher for targets occurring at the long foreperiod, thus explaining the decrease in $\mathrm{RT}$ observed with long foreperiod trials of variable foreperiod studies.

The study by Vallesi, Lozano, and Correa (2013) will be used to explain how to measure the variable foreperiod effect. Here, we will consider only the 
data from the blocks with a short inter-trial interval (ITI) of Experiment 2 (simple-RT task). An adapted version of the original experiment can be found in book's GitHub repository.

In the study by Vallesi et al. (2013), the variable foreperiod design included an auditory warning signal (a $1500 \mathrm{~Hz}$ pure tone) and a visual target (a square or a triangle) with a foreperiod of either 1000 or $3000 \mathrm{~ms}$. Each foreperiod was presented randomly with the same probability. A yellow cross, which served as the fixation point, was presented simultaneously with the warning signal and remained on the screen until the target appeared. The participants' task was to respond to the onset of the target by pressing the space bar with their index finger (see Figure 10.1A).

As can be seen in Figure 10.1B, participants' RTs were shorter for the long foreperiod than for the short foreperiod. Such a difference reflects the foreperiod effect typically found in a variable foreperiod design. Several studies have replicated the foreperiod effect across different foreperiod ranges and type of tasks (Niemi \& Näätänen, 1981). Hence, an interim conclusion from this phenomenon is that our cognitive system can exploit the temporal information implicitly provided by task context to improve performance.

Another type of phenomenon that further proves the increase in sensitivity to events having predictable timing is generally termed "sequential effects" (e.g., Capizzi, Correa, Wojtowicz, \& Rafal, 2015; Los \& van den Heuvel, 2001; Los, 2013; Steinborn \& Langner, 2012; Steinborn, Rolke, Bratzke, \& Ulrich, 2008; Vallesi \& Shallice, 2007; Woodrow, 1914). Sequential effects take into account the influence that the previous foreperiod duration has on preparation in the current foreperiod. Thus, following the variable foreperiod procedure, the analysis should now consider whether the previous foreperiod was short or long. To illustrate this point, we turn back to the experiment by Vallesi et al. (2013). When results are analyzed considering the previous foreperiod as a factor (pre-Short vs. pre-Long), the pattern usually conforms to the one depicted in Figure 10.1C. As shown in this figure, participants' RTs were overall shorter when the previous foreperiod was short rather than long. More importantly, a significant interaction between previous foreperiod and current foreperiod duration was also observed. This interaction reflects the "asymmetry" of sequential effects. That is, on current short foreperiod trials participants' responses were faster after a previous short foreperiod as compared to alternation from a previous long foreperiod (in Figure $10.1 \mathrm{C}$, the two conditions are associated with 316 and $361 \mathrm{~ms}$, respectively). Conversely, on current long foreperiod trials, participants' responses were equally fast irrespective of whether the previous foreperiod duration had been short or long (in Figure 10.1C, $305 \mathrm{~ms}$ for both conditions). 
A)

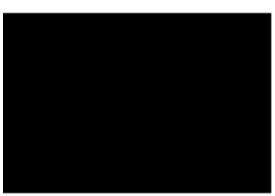

$1000 \mathrm{~ms}$
Warning signal

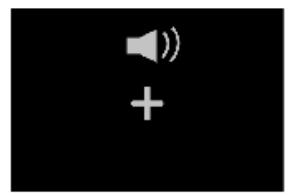

$50 \mathrm{~ms}$
Foreperiod

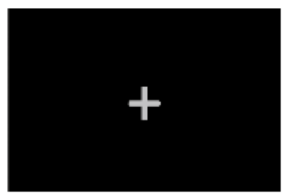

950 or $2950 \mathrm{~ms}$
Target

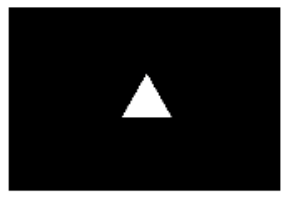

$300 \mathrm{~ms}$



C)

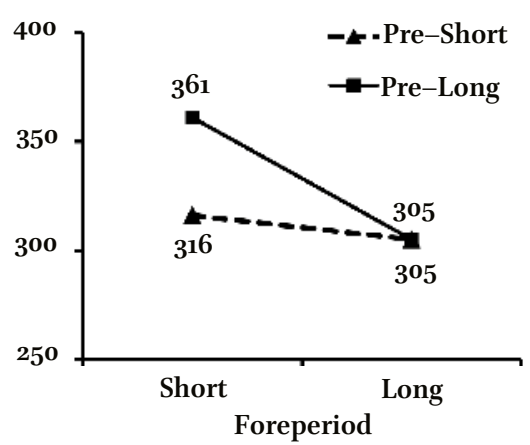

FIGURE 10.1 (A) Experimental design. The variable foreperiod paradigm used by Vallesi, Lozano, and Correa (2013). In the original study, the cross was displayed in yellow instead of gray. Each trial started with the ITI: a black screen lasting $1000 \mathrm{~ms}$. Subsequently, the auditory warning signal and the fixation cross were presented. The fixation cross remained on the screen for a variable foreperiod interval of 1000 or $3000 \mathrm{~ms}$. Then, the target (a square or a triangle) appeared and participants had to respond to it by pressing the space bar. The target disappeared after either the participants' response or after $1.500 \mathrm{~ms}$. (B) The foreperiod effect. Participants' RTs in ms are plotted as a function of the Foreperiod duration (short, long). The foreperiod effect is indexed by shorter RTs at the long foreperiod. (C) Sequential effects. Participants' RTs are plotted as a function of both the previous foreperiod duration (label Pre-in the figure legend) and the current foreperiod duration. The asymmetry of sequential effects is given by shorter RTs for the short-short foreperiod sequence as compared to the long-short foreperiod sequence. Procedure and data for both the foreperiod and sequential effects are adapted from Vallesi, Lozano and Correa (2013).

Since the first demonstration of the presence of sequential effects in temporal preparation (Woodrow, 1914), there have been several replications suggesting that such effects are quite robust. A comprehensive overview of the cognitive models that have been developed to explain sequential effects is beyond the scope of this chapter (for further information, see Capizzi et al., 2015; Los \& van den Heuvel, 2001; Steinborn et al., 2008; Vallesi \& Shallice, 2007, and the following book chapters: Los, 2010; Vallesi, 2010). 
A general agreement exists that sequential effects, unlike the foreperiod effect, are mediated by automatic processes. This claim is bolstered by converging evidence provided by behavioral (Vallesi, Arbula, \& Bernardis, 2014; Vallesi et al., 2013), neuropsychological (Triviño, Correa, Arnedo, \& Lupiáñez, 2010; Vallesi et al., 2007a), developmental (Vallesi \& Shallice, 2007), and transcranial magnetic stimulation (Vallesi, Shallice, \& Walsh, 2007b) studies, which dissociated foreperiod and sequential effects. For example, the foreperiod effect only has been shown to rely on the functioning of prefrontal structures related to executive processes, whereas it has been shown not to be the case for sequential effects (Triviño et al., 2010; Vallesi et al., 2007a). These dissociations demonstrate that temporal preparation can be flexibly built up on the basis of distinct controlled and automatic processes. A clear example of a controlled process of temporal preparation is given by "temporal orienting of attention", which is the focus of the next section.

Coull and Nobre (1998) developed a temporal variant of the Posner's spatial orienting task (Posner, Snyder, \& Davidson, 1980) to test whether attention can be voluntarily oriented in time. In a typical implementation of the temporal orienting task (see Correa, 2010), participants have to respond to the onset of a target stimulus that can appear either early in time (e.g., after a short foreperiod of $1000 \mathrm{~ms}$ ) or late in time (e.g., after a long foreperiod of $3000 \mathrm{~ms}$ ). A symbolic cue (e.g., a short or a long line) precedes each target presentation indicating whether the target is likely to appear early or late, respectively. On a large percentage of trials (e.g., $75 \%$ ), the cue validly predicts the length of the foreperiod (valid condition). On the remaining trials, the target will appear either earlier or later than expected (invalid condition). As shown in Figure 10.2B, this design gives rise to four types of trials. The 'early cue-short foreperiod' and the 'late cue-long foreperiod' types of trials are valid, whereas the 'early cue-long foreperiod' ('delayed', cf. Coull, 2011) and the 'late cue-short foreperiod' ('premature') trials are invalid.

Figure 10.2 depicts the temporal orienting task used by Capizzi, Sanabria and Correa (2012) and their results, that is, mean RTs from the single-task condition plotted as a function of Cue Validity (valid, invalid) and Foreperiod (short, long). An adapted version of the original experiment can be downloaded from the book's GitHub repository.

As shown in Figure $10.2 \mathrm{C}$, participants' RTs were shorter for valid trials as compared to invalid trials. This finding indexes the temporal orienting (or 
A) Blank

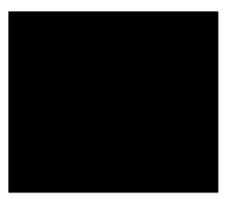

500-1000 ms
Cue



$750 \mathrm{~ms}$

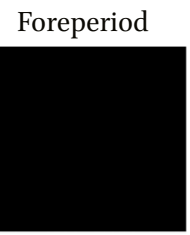

1000 or $3000 \mathrm{~ms}$

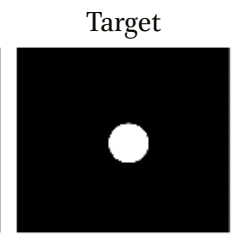

$100 \mathrm{~ms}$

Blank

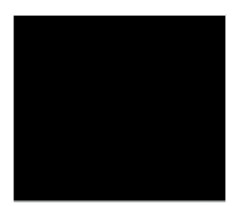

Until response or $1100 \mathrm{~ms}$

Time

C)

\begin{tabular}{c|cc}
\hline \multirow{2}{*}{ CUE } & \multicolumn{2}{|c}{ FOREPERIOD } \\
& Short & Long \\
\hline Early & $\begin{array}{c}\text { Valid trial } \\
(75 \%)\end{array}$ & $\begin{array}{c}\text { Invalid trial } \\
\text { (Delayed) }\end{array}$ \\
Late & $\begin{array}{c}\text { Invalid trial } \\
(\text { Premature })\end{array}$ & $\begin{array}{c}\text { Valid trial } \\
(75 \%)\end{array}$ \\
\hline
\end{tabular}

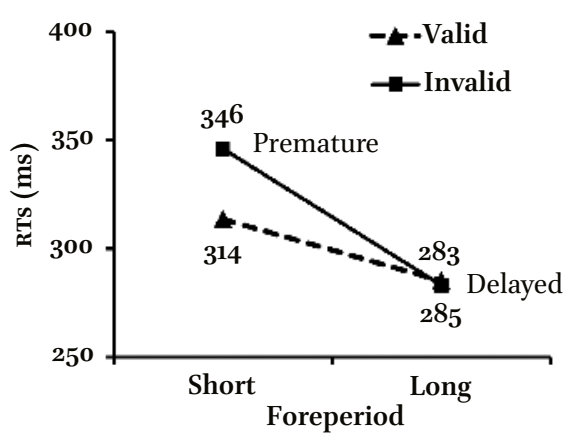

FIGURE 10.2 (A) Experimental design. The temporal orienting task used by Capizzi, Sanabria, and Correa (2012). In the original study, the cue (here displayed in gray) could be filled with one of three colors (red, green, or blue). Each trial started with the presentation of a blank screen for a random interval between 500 and $1000 \mathrm{~ms}$. The temporal cue (a short or a long line) was then displayed for $750 \mathrm{~ms}$. The short line indicated that the target was likely to appear early (after a short foreperiod), whereas the long line indicated that the target would probably appear late (after a long foreperiod). Following the cue, the screen remained blank for a variable foreperiod of 1000 or $3000 \mathrm{~ms}$. After the foreperiod elapsed, the target stimulus (a white dot) appeared in the center of the screen for a duration of $100 \mathrm{~ms}$. Participants had to respond to the target onset by pressing the space bar with their index finger. The next trial began following the response to the target or after 1100 ms. (B) Types of trials. The matching between early cue and short foreperiod and between late cue and long foreperiod gives rise to valid trials ( $75 \%$ validity rate). Conversely, the combination of early cue and long foreperiod produces invalid 'delayed' trials since the target appears later than expected. Lastly, the combination of late cue and short foreperiod creates invalid 'premature' trials in which the target appears earlier than expected. (C) Temporal orienting effects. Participants' RTs are plotted as a function of Cue Validity (valid, invalid) and Foreperiod duration (short, long). Note that temporal orienting effects are significant at the short foreperiod only. This is due to the fact that when the target does not show up after the short foreperiod (as invalidly predicted by the early cue), participants can re-orient their attention to the long foreperiod, thus counteracting the negative consequences on performance of the invalid prediction. The procedure and data are adapted from Capizzi et al. (2012). 
validity) effects. These effects are usually restricted to the short foreperiod only, as demonstrated by the interaction between Cue Validity and Foreperiod. Turning to the figure, a RT benefit can be observed for validly early cued targets (early cue-short foreperiod) as compared to 'premature' targets that invalidly occur earlier than expected (late cue-short foreperiod). By contrast, at the long foreperiod, temporal orienting effects are smaller (or can even be absent). This is because if the target does not appear at the short foreperiod as predicted by the early cue, it is possible to 'infer' that it will appear later, thus enabling attention to be re-oriented to the long foreperiod (invalid 'delayed' trials in the figure) (e.g., Correa, Lupiáñez, Milliken, \& Tudela, 2004; Coull \& Nobre, 1998).

In the remainder of the section, we list some practical issues that should be considered when designing a temporal orienting task. One issue concerns the cue validity rate. Most studies use cue validity rates of 75 or $80 \%$. However, it is also possible to use a $100 \%$ valid cue condition and to compare it with a neutral one in which the cue does not provide any temporal information on target onset. That is, in the neutral condition the cue simply acts as a warning signal and the target can appear after the short or long foreperiod with the same frequency $(50 \%)$. Use of valid-only trials encourages participants to confidently rely on the cue information. This manipulation has been adopted in studies investigating the ability to orient attention in time in more vulnerable populations such as children (Mento \& Tarantino, 2015; Mento \& Vallesi, 2016) and older adults (Zanto et al., 2011).

If a researcher is interested in maximizing temporal orienting effects, it is possible to keep 'early' and 'late' temporal cues separate across different blocks of trials instead of intermixing them within trials. In that case, participants will be presented with early-blocks and late-blocks, each containing both valid and invalid trials. Specifically, the early-block will only display the cue type assigned to the early condition. On $75 \%$ of the trials, the foreperiod will match the duration predicted by the cue, whereas on $25 \%$ of the trials it will not. The same applies, but with the late cue-long foreperiod association, to the lateblock condition. It is important to counterbalance the order of presentation of early and late blocks to avoid confounding any practice or boredom effects with one of the two conditions. In previous studies, we have compared betweenblock and within-block manipulations of temporal cues and found that the magnitude of temporal orienting effects were larger in the former case (Correa, Lupiáñez, \& Tudela, 2006b). Moreover, we recently reported that temporal orienting effects elicited on a trial-by-trial basis, but not those obtained in a blocked design, were impaired in dual-task conditions that competed for common limited resources with the temporal orienting task (Capizzi et al., 2012). 
A blocked manipulation of temporal cues is, thus, advisable when evaluating temporal orienting in populations that get tired and unmotivated after brief periods of time, such as frontal brain damaged patients who, notoriously, have troubles maintaining the focus of attention on the task at hand (see Triviño et al., 2011; Triviño et al., 2010).

Another factor that can affect the magnitude of temporal orienting effects includes cue encoding. It is recommended to employ intuitive cues that do not need a demanding and time-consuming decoding process. Some examples of previously used intuitive cues comprise symbolic figures: two concentric circles with the inner circle brightening for the short foreperiod and the outer circle for the long one (Coull \& Nobre, 1998) or short and long lines (Capizzi, Correa, \& Sanabria, 2013; Capizzi et al., 2012; Correa et al., 2014; Correa et al., 2004; Correa, Lupiáñez, \& Tudela, 2006b; Triviño et al., 2010); letters: 'S' for short, 'L' for long (Zanto et al., 2011) and words: 'early' and 'late' (Correa, Lupiáñez, Madrid, \& Tudela, 2006a).

To strengthen temporal orienting effects, it is also important to provide participants with enough practice trials to initially learn the specific cue-time interval contingencies. Moreover, participants' instructions should explicitly emphasize that using the temporal cue would help them to predict when the target will occur.

Finally, the type of task also influences temporal orienting effects. We have shown, for example, that temporal orienting effects are usually larger in a simple-RT detection task than in a choice-RT discrimination task (Correa et al., 2004; Correa, Lupiáñez, \& Tudela, 2006b). However, only the latter, which imposes strong demands on perceptual analysis, boosts visual processing as revealed by event-related potential (ERP) measures. Otherwise, in simple RTdetection tasks, temporal orienting typically enhances performance through facilitation of late motor processes (see Correa, Lupiáñez, Madrid, \& Tudela, 2006a, for a review). Of note, more sophisticated tasks than the originally employed detection and discrimination ones have been developed over the last years to elucidate whether temporal orienting effects can also act on processes requiring higher cognitive functioning. These studies showed that temporal orienting can modulate semantic processing (Naccache, Blandin, \& Dehaene, 2002), executive control (Correa, Cappucci, Nobre, \& Lupiáñez, 2010a), and visual working memory (van Ede, Niklaus, \& Nobre, 2017), thus demonstrating that temporal orienting effects are robust across different tasks and cognitive demands.

The majority of the earlier studies investigating temporal orienting have used symbolic and static cues as a means of orienting attention in time. 
However, since we live in a dynamic environment, which is intrinsically rhythmic, we should also be able to generate temporal expectations following the regular presentation of isochronous sequences of stimuli (i.e., rhythms). This topic will be covered in the following section.

According to the Dynamic Attending Theory (Ellis \& Jones, 2010; Large \& Jones, 1999), attention is conceived of as an endogenous oscillatory process that can be entrained by external rhythms. The repetition of a regular rhythmic pattern will, thus, synchronize participants' attentional oscillations, which improves both accuracy and response speed to a target appearing in synchrony with that pattern (e.g., Bolger, Coull, \& Schön, 2014; Bolger, Trost, \& Schön, 2013; Breska \& Deouell, 2014; Di Luca \& Rhodes, 2016; Jones et al., 2002; Lange, 2010; Rohenkohl, Cravo, Wyart, \& Nobre, 2012; Sanabria et al., 2011; Sanabria \& Correa, 2013). It is important to note that unlike the temporal orienting effects described above, rhythms can automatically orient attention in time. For example, participants' performance is entrained by the rhythms even if they are instructed to ignore them (Rohenkohl, Coull, \& Nobre, 2011). The automaticity of temporal preparation driven by auditory rhythms has been tested in dual-task studies that showed no decrement in performance on rhythm-based preparation in the context of a concurrent memory task (Cutanda et al., 2015; de la Rosa, Sanabria, Capizzi, \& Correa, 2012).

An example of how to investigate temporal preparation driven by auditory rhythms can be found in de la Rosa et al. (2012). In this chapter, we will only focus on the single-task data of Experiment 1. An adapted version of the original experiment can be downloaded from the book's GitHub repository. As shown in Figure 10.3A, the task was comprised of a sequence of six tones with a 250-ms duration each and a frequency of $700 \mathrm{~Hz}$. This sequence could be temporally regular or irregular. The regular sequence was created by spacing the tones with a fixed interval of $55^{\circ} \mathrm{ms}$. Conversely, in the irregular rhythm each interval duration was selected randomly among the following values: $150,35^{\circ}, 55^{\circ}, 75^{\circ}$, or $95^{0} \mathrm{~ms}$. Importantly, both sequences included six tones and had identical duration such that the only difference between the two concerned the isochrony of the rhythm. The target tone, which appeared after a variable foreperiod (800, 1100, or $1400 \mathrm{~ms})$, was a 100-ms sound of $400 \mathrm{~Hz}$. Participants had to respond to the target tone by pressing the "b" key on a computer keyboard. They were informed that the target was preceded by a sequence of sounds creating a rhythm. However, 


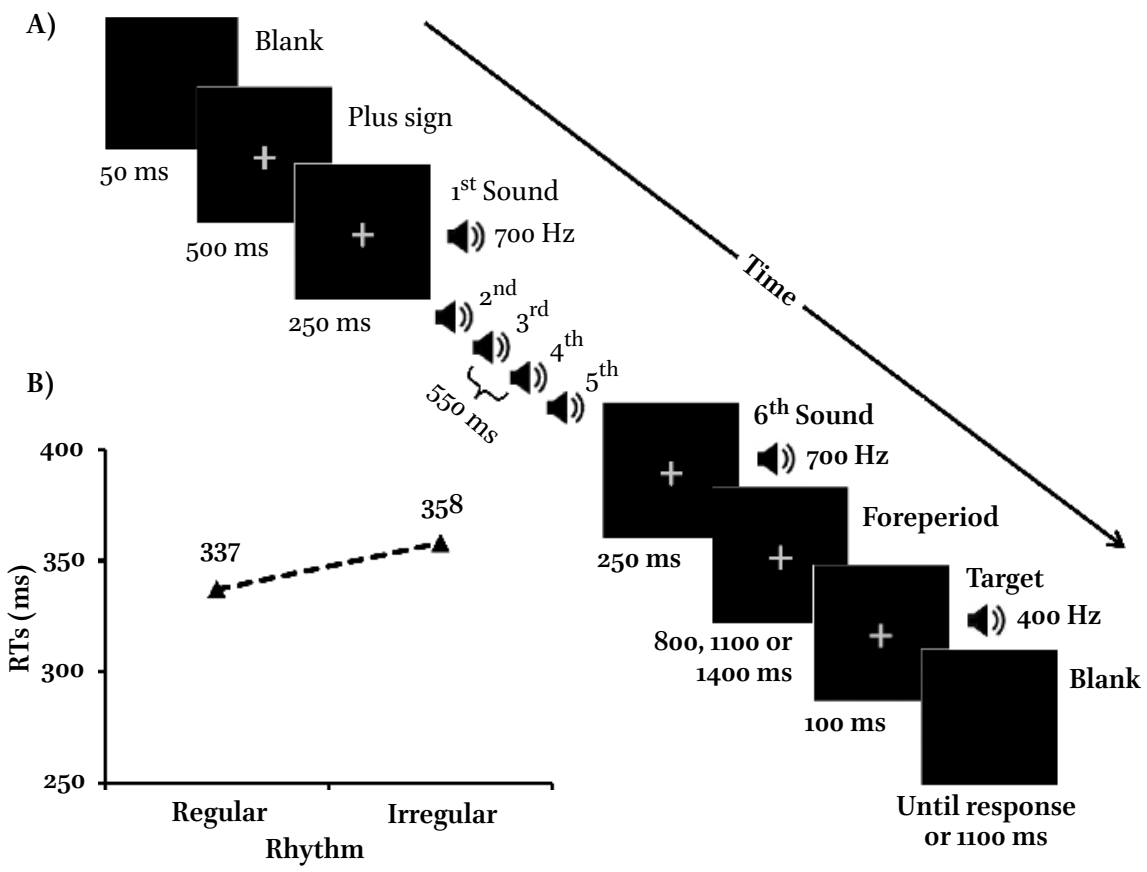

FIGURE 10.3 (A) Experimental design. The rhythm-based temporal preparation task used by de la Rosa, Sanabria, Capizzi, and Correa (2012). In the original study, the fixation cross (a plus sign, here displayed in gray), which remained on the screen during the entire trial, could be filled with one of three colors (red, green, or blue). Each trial started with the presentation of a 50 -ms blank screen. Five hundred milliseconds later, a regular or irregular rhythm was presented at random. To create the regular rhythm, as shown in the figure, all the intervals between the six tones had a fixed duration of $550 \mathrm{~ms}$. Conversely, in the irregular rhythm each interval duration was selected randomly among the following values: 150, 350, 550,750 , or $950 \mathrm{~ms}$. After a variable foreperiod (800, 1100, or $1400 \mathrm{~ms}$ ), the target tone (a $400-\mathrm{Hz}$ sound lasting for $100 \mathrm{~ms}$ ) was presented. Participants had to press the " $b$ " key as fast as possible to the onset of the target tone. The next trial began following the response to the target, or after $1100 \mathrm{~ms}$. (B) Regularity effects. Participants' RTs are plotted as a function of Rhythm (regular, irregular). In line with the hypothesis that the presentation of a regular rhythm improves performance, participants were faster in responding to targets appearing after a regular rhythm compared to an irregular rhythm. The procedure and data are adapted from de la Rosa et al. (2012).

they were explicitly told that this rhythm had no bearing on the task and could, therefore, be ignored.


lar, irregular). As depicted, participants were faster to detect the target that 
appeared after the regular rhythmic pattern compared to the target preceded by an irregular sequence. This demonstrates that it is possible to develop temporal preparation on the basis of regular auditory rhythms. Please note that rhythms represent a powerful way of orienting attention in time also in the visual modality. Some examples of paradigms that have employed visual rhythms can be found in the following studies (Correa and Nobre, 2008; Correa et al., 2014; Doherty, Rao, Mesulam, \& Nobre, 2005; Triviño et al., 2011).

As a last note, it is worth mentioning that in addition to non-predictive (regular vs. irregular) rhythms (e.g., de la Rosa et al., 2010; Lange, 2010), it is also possible to employ predictive rhythms to induce temporal preparation. This can be done, for example, by manipulating the pace (fast or slow) of the rhythmic pattern (e.g., Correa et al., 2014; Triviño et al., 2011). That is, a fast rhythmic pace predicts that the target would appear early, whereas a slow rhythmic pace predicts that the target would appear late. A rhythm-based benefit is also observed using predictive rhythms. However, it is difficult to ascertain whether this advantage is due to the exogenous effects of rhythms alone, or also to other endogenous processes sensitive to the temporal contingency between the rhythmic pace and the moment of target onset, or to a mixture of both factors. It is, thus, important to take into account these features of the experimental design when thinking of how to investigate rhythmbased preparation.

\section{Conclusions and Practical Considerations}

In this chapter, we provided a general overview of the typical experimental procedures developed to measure temporal preparation. This conclusive section offers some final advice that may help determine the best procedure to use for addressing a given research question. To this aim, we start by briefly considering the relationship between different temporal preparation phenomena (see Correa, 2010, for a theoretical discussion on this issue). If, for example, the focus of the study is measuring temporal orienting, foreperiod, and sequential effects within the same task, there are no disadvantages to using a temporal orienting paradigm. Correa and colleagues (Triviño et al., 2010; see also Correa et al., 2010b) developed an adapted and quick version of the temporal orienting task that allows the evaluation of the three temporal preparation effects at the same time. By contrast, if one is interested in investigating the ERP correlates of both temporal orienting and sequential effects, it is advisable to also have a neutral condition in which the temporal cue is substituted with 
a non-informative warning signal. Otherwise, it has been suggested that the ERP correlates of sequential effects may be masked by the controlled temporal orienting processing (Capizzi et al., 2013).

Another example of the reciprocal influence between different temporal preparation effects can be found in Sanabria, Capizzi, and Correa (2011). The authors showed that temporal preparation driven by auditory rhythms was effective at the long foreperiod only when catch-trials (i.e., trials in which a target is not presented) were included. This is due to the fact that when the target always occurs, the certainty that it will appear at the long foreperiod, once the short foreperiod has been passed, is so strong that it overcomes the benefits afforded by the auditory rhythms. The presence of catch-trials instead counteracts the foreperiod effect by decreasing participants' certainty about target occurrence (Näätänen, 1972). Note, also, that the inclusion of catch-trials has been useful to unveil the effects of both temporal orienting (Correa et al., 2004) and sequential effects (Capizzi et al., 2015) at the long foreperiod.

Once the best approach to use has been established, it is critical to adapt the chosen paradigm according to the specific target population. As an illustrative example, Mento and colleagues (Mento \& Tarantino, 2015; Mento \& Vallesi, 2016) have recently developed a simplified child-friendly version of the temporal orienting paradigm. In this version, children imagine being at the zoo and taking pictures of the animals that appear within the camera lens. They are instructed that the color of the lens will help them to predict when the animal will appear.

Other examples of research that have been fruitfully conducted in special populations can be found in the following studies (e.g., Droit-Volet \& Coull, 2016; Johnson et al., 2016; Johnson, Burrowes, \& Coull, 2015; Mento \& Valenza, 2016; Vallesi \& Shallice, 2007, for children; Bherer \& Belleville, 2004; Chauvin et al., 2016; Vallesi, McIntosh, \& Stuss, 2009b; Zanto et al., 2011, for older adults; Correa et al., 2010b, for individuals with high and low trait impulsivity; Marzecova et al., 2013, for bilinguals). Moreover, the reader interested in investigating temporal preparation in clinical settings can refer to these studies (Triviño et al., 2016, 2011, 2010; Vallesi et al., 2007a, for neurological patients; Correa et al., 2011, for patients with fibromyalgia).

To conclude, the increasing number of studies that have investigated temporal preparation across different tasks and populations demonstrates the growing interest in this area of research. Future developments will, thus, include devising novel experimental designs and, importantly, linking the theory of temporal preparation to practice-oriented research. 


\section{Acknowledgements}

M.C. was supported by a post-doctoral fellowship from the European Research Council Starting grant $\mathrm{n}^{\circ} 313692$ ( $\left.\mathrm{FP}_{7} / 2007-2013\right)$. A.c. was supported by the Junta de Andalucía (Proyectos de Excelencia: SEJ-3054).

\section{References}

Bausenhart, K.M., B. Rolke, \& R. Ulrich (2008). Temporal preparation improves temporal resolution: Evidence from constant foreperiods. Perception \& Psychophysics, $70(8), 1504-1514$.

Bherer, L., \& S. Belleville (2004). Age-related differences in response preparation: The role of time uncertainty. Journal of Gerontology B: Psychology Science and Social Science, 59, 66-74.

Bolger, D., J.T. Coull, \& D. Schön (2014). Metrical rhythm implicitly orients attention in time as indexed by improved target detection and left inferior parietal activation. Journal of Cognitive Neuroscience, 26(3), 593-605.

Bolger, D., W. Trost, \& D. Schön (2013). Rhythm implicitly affects temporal orienting of attention across modalities. Acta Psychologica, 142(2), 238-244.

Breska, A., \& L.Y. Deouell (2014). Automatic bias of temporal expectations following temporally regular input independently of high-level temporal expectation.Journal of Cognitive Neuroscience, 26(7), 1555-1571.

Capizzi, M., A. Correa, \& D. Sanabria (2013). Temporal orienting of attention is interfered by concurrent working memory updating. Neuropsychologia, 51(2), 326-339.

Capizzi, M., A. Correa, A. Wojtowicz, \& R.D. Rafal (2015). Foreperiod priming in temporal preparation: Testing current models of sequential effects. Cognition, 134, 39-49.

Capizzi, M., D. Sanabria, \& A. Correa (2012). Dissociating controlled from automatic processing in temporal preparation. Cognition, 123(2), 293-302.

Chauvin, J.J., C.R. Gillebert, G. Rohenkohl, G.W. Humphreys, \& A.C. Nobre (2016). Temporal orienting of attention can be preserved in normal aging. Psychology and aging, 31(5), 442-455.

Correa, A. (2010). Enhancing behavioural performance by visual temporal orienting. In Nobre, A.C. and J.T. Coull (Eds.), Attention and time (pp. 357-370). Oxford University Press.

Correa, A., P. Cappucci, A.C. Nobre, \& J. Lupiáñez (2010a). The two sides of temporal orienting: Facilitating perceptual selection, disrupting response selection. Experimental Psychology, 57(2), 142-148. 
Correa, A., G. Cona, S. Arbula, A. Vallesi, \& P. Bisiacchi (2014). Neural dissociation of automatic and controlled temporal preparation by transcranial magnetic stimulation. Neuropsychologia, 65, 131-136.

Correa, A., J. Lupiáñez, E. Madrid, \& P. Tudela (2006a). Temporal attention enhances early visual processing: A review and new evidence from event-related potentials. Brain Research, 1076(1), 116-128.

Correa, A., J. Lupiáñez, B. Milliken, \& P. Tudela (2004). Endogenous temporal orienting of attention in detection and discrimination tasks. Perception \& Psychophysics, $66(2), 264-278$.

Correa, A., J. Lupiáñez, \& P. Tudela (2006b). The attentional mechanism of temporal orienting: Determinants and attributes. Experimental Brain Research, 169(1), 58-68.

Correa, A., E. Miró, M.P. Martínez, A.I. Sánchez, \& J. Lupiáñez (2011). Temporal preparation and inhibitory deficit in fibromyalgia syndrome. Brain and Cognition, 75(3), 211-216.

Correa, A., \& A.C. Nobre (2008). Neural modulation by regularity and passage of time. Journal of Neurophysiology, $100(3), 1649-1655$.

Correa, A., M. Triviño, C. Pérez-Dueñas, A. Acosta, \& J. Lupiañez (2010b). Temporal preparation, response inhibition and impulsivity. Brain and Cognition, 73(3), 222-228.

Coull, J.T. (2011). Discrete neuroanatomical substrates for generating and updating temporal expectations. In Dehaene, S. \& E. Brannon (eds.), Time and Number in the Brain: Searching for the Foundations of Mathematical Thought. Elsevier.

Coull, J.T. (2009). Neural substrates of mounting temporal expectation. PLoSBiology, $7(8)$, e1000166.

Coull, J.T., \& A.C. Nobre (1998). Where and when to pay attention: the neural systems for directing attention to spatial locations and to time intervals as revealed by both pet and fMRI. Journal of Neuroscience, 18(18), 7426-7435.

Cutanda, D., A. Correa, \& D. Sanabria (2015). Auditory temporal preparation induced by rhythmic cues during concurrent auditory working memory tasks. Journal of Experimental Psychology. Human Perception and Performance, 41(3), 790-797.

de la Rosa, M.D., D. Sanabria, M. Capizzi, \& A. Correa (2012). Temporal preparation driven by rhythms is resistant to working memory interference. Frontiers in Psychology, 3, 308 .

Di Luca, M., \& D. Rhodes (2016). Optimal perceived timing: Integrating sensory information with dynamically updated expectations. Scientific Reports, 6, 28563.

Doherty, J.R., A. Rao, M.M. Mesulam, \& A.C. Nobre (2005). Synergistic effect of combined temporal and spatial expectations on visual attention. Journal of Neuroscienc, $25(36), 8259-8266$. 
Drazin, D.H. (1961). Effects of foreperiod, foreperiod variability, and probability of stimulus occurrence on simple reaction time. Journal of Experimental Psychology, $62,43-50$.

Droit-Volet, S., \& J.T. Coull (2016). Distinct developmental trajectories for explicit and implicit timing. Journal of Experimental Child Psychology, 150, 141-154.

Elithorn, A., \& C. Lawrence (1955). Central inhibition: Some refractory observations. Quarterly Journal of Experimental Psychology, 11, 211-220.

Ellis, R.J., \& M.R. Jones (2010). Rhythmic context modulates foreperiod effects. Attention, Perception \& Psychophysics, 72(8), 2274-2288.

Gibbon, J. (1977). Scalar expectancy theory and Weber's law in animal timing. Pychological Review, 84, 279-385.

Grondin, S. (2010). Timing and time perception: A review of recent behavioral and neuroscience findings and theoretical directions. Attention, Perception \& Psychophysics, $72(3), 5^{61-582 .}$

Ivry, R.B., \& R.E. Hazeltine (1995). Perception and production of temporal intervals across a range of durations: Evidence for a common timing mechanism. Journal of Experimental Psychology. Human Perception and Performance, 21(1), 3-18.

Johnson, K.A., M. Bryan, K. Polonowita, D. Decroupet, \& J.T. Coull (2016). Isochronous sequential presentation helps children orient their attention in time. Frontiers in Psychology, 7, 1417.

Johnson, K.A., E. Burrowes, \& J.T. Coull (2015). Children can implicitly, but not voluntarily, direct attention in time. PloS One, $10(4)$, eo123625.

Jones, M.R., H. Moynihan, N. MacKenzie, \& J. Puente (2002). Temporal aspects of stimulus-driven attending in dynamic arrays. Psychological Science, 13(4), 313-319.

Klemmer, E.T. (1956). Time uncertainty in simple reaction time. Journal of Experimental Psychology, 51(3), 179-184.

Klemmer, E.T. (1957). Simple reaction time as a function of time uncertainty. Journal of Experimental Psychology, 54(3), 195-200.

Lange, K. (2010). Can a regular context induce temporal orienting to a target sound? International Journal of Psychophysiology: Official Journal of the International Organization of Psychophysiology, 78(3), 231-238.

Large, E.W., \& M.R. Jones (1999). The dynamics of attending: How people track timevarying events. Psychological Review, 106(1), 119-159.

Los, S.A. (2010). Foreperiod and the sequential effect: Theory and data. In Nobre, A.C. \& J.T. Coull (Eds.), Attention and time (pp. 289-302). Oxford: Oxford University Press.

Los, S.A. (2013). The role of response inhibition in temporal preparation: Evidence from a go/no-go task. Cognition, 129(2), 328-344.

Los, S.A., \& C.E. van den Heuvel (2001). Intentional and unintentional contributions to nonspecific preparation during reaction time foreperiods. Journal of Experimental Psychology. Human Perception and Performance, 27(2), 370-386. 
Marzecova, A., M. Bukowski, A. Correa, M. Boroso, J. Lupiañez, \& Z. Wodniecka (2013). Tracing the bilingual advantage in cognitive control:The role of flexibility in temporal preparation and category switching. Journal of Cognitive Psychology, 25, 586-604.

Mattes, S., \& R. Ulrich (1997). Response force is sensitive to the temporal uncertainty of response stimuli. Perception \& Psychophysics, 59(7), 1089-1097.

Mento, G., \& V. Tarantino (2015). Developmental trajectories of internally and externally driven temporal prediction. PLoS ONE, $10(8), 1-18$.

Mento, G., V. Tarantino, A. Vallesi, \& P.S. Bisiacchi (2015). Spatiotemporal neurodynamics underlying internally and externally driven temporal prediction: A high spatial resolution ERP study. Journal of Cognitive Neuroscience, 27(3), 425-439.

Mento, G., \& E. Valenza (2016). Spatiotemporal neurodynamics of automatic temporal expectancy in 9-month old infants. Scientific Reports, 6, 36525 .

Mento, G., \& A. Vallesi (2016). Spatiotemporally dissociable neural signatures for generating and updating expectation over time in children: A High Density-ERP study. Developmental Cognitive Neuroscience, 19, 98-106.

Miniussi, C., E.L. Wilding, J.T. Coull, \& A.C. Nobre (1999). Orienting attention in time: Modulation of brain potentials. Brain, 122, 1507-1518.

Näätänen, R. (1972). Time uncertainty and occurrence uncertainty of the stimulus in a simple reaction time task. Acta Psychologica, 36, 492-503.

Naccache, L., E. Blandin, \& S. Dehaene (2002). Unconscious masked priming depends on temporal attention. Psychological Science, 13(5), 416-424.

Niemi, P., \& R. Näätänen (1981). Foreperiod and simple reaction time. Psychological Bulletin, 89(1), 133-162.

Nobre, A.C. (2001). Orienting attention to instants in time. Neuropsychologia, 39, $1317-1328$.

Nobre, A.C., A. Correa, \& J.T. Coull (2007). The hazards of time. Current Opinion in Neurobiology, 17(4), 465-470.

Nobre, A.C., \& J.T. Coull (2010). Attention and time. Oxford: Oxford University Press.

Posner, M.I., C.R. Snyder, \& B.J. Davidson (1980). Attention and the detection of signals. Journal of Experimental Psychology, 109(2), 160-174.

Rohenkohl, G., J.T. Coull, \& A.C. Nobre (2011). Behavioural dissociation between exogenous and endogenous temporal orienting of attention. PLoS ONE, 6(1), 1-5.

Rohenkohl, G., A.M. Cravo, V. Wyart, \& A.C. Nobre (2012). Temporal expectation improves the quality of sensory information.Journal of Neuroscience 32(24), 8424-8428.

Sanabria, D., M. Capizzi, \& A. Correa (2011). Rhythms that speed you up. Journal of Experimental Psychology. Human Perception and Performance, 37(1), 236-244.

Sanabria, D., \& A. Correa (2013). Electrophysiological evidence of temporal preparation driven by rhythms in audition. Biological Psychology, 92(2), 98-105.

Steinborn, M.B., \& R. Langner (2012). Arousal modulates temporal preparation under increased time uncertainty: Evidence from higher-order sequential foreperiod effects. Acta Psychologica, 139(1), 65-76. 
Steinborn, M.B., B. Rolke, D. Bratzke, \& R. Ulrich (2008). Sequential effects within a short foreperiod context: Evidence for the conditioning account of temporal preparation. Acta Psychologica, 129(2), 297-307.

Triviño, M., M. Arnedo, J. Lupiáñez, J. Chirivella, \& A. Correa (2011). Rhythms can overcome temporal orienting deficit after right frontal damage. Neuropsychologia, 49(14), 3917-3930.

Triviño, M., A. Correa, M. Arnedo, \& J. Lupiáñez (2010). Temporal orienting deficit after prefrontal damage. Brain, 133(4), 1173-1185.

Triviño, M., A. Correa, J. Lupiáñez, M.J. Funes, A. Catena, X. He, \& G.W. Humphreys (2016). Brain networks of temporal preparation: A multiple regression analysis of neuropsychological data. NeuroImage, 142, 489-497.

Vallesi, A. (2010). Neuroanatomical substrates of foreperiod effects. In Nobre, A.C. \& J.T. Coull (Eds.), Attention and time (pp. 303-316). Oxford: Oxford University Press.

Vallesi, A., S. Arbula, \& P. Bernardis (2014). Functional dissociations in temporal preparation: evidence from dual-task performance. Cognition, 130(2), 141-151.

Vallesi, A., V.N. Lozano, \& A. Correa (2013). Dissociating temporal preparation processes as a function of the inter-trial interval duration. Cognition, 127(1), 22-30.

Vallesi, A., A.R. McIntosh, T. Shallice, \& D.T. Stuss (2009a). When time shapes behavior: fMRI evidence of brain correlates of temporal monitoring. Journal of Cognitive Neuroscience, $27(6), 1116-1126$.

Vallesi, A., A.R. McIntosh, \& D.T. Stuss (2009b). Temporal preparation in aging: A functional MRI study. Neuropsychologia, 47(13), 2876-2881.

Vallesi, A., A. Mussoni, M. Mondani, R. Budai, M. Skrap, \& T. Shallice (2007a). The neural basis of temporal preparation: Insights from brain tumor patients. Neuropsychologia, 45(12), 2755-2763.

Vallesi, A., \& T. Shallice (2007). Developmental dissociations of preparation over time: Deconstructing the variable foreperiod phenomena. Journal of Experimental Psychology. Human Perception and Performance, 33(6), 1377-1388.

Vallesi, A., T. Shallice, \& V. Walsh (2007b). Role of the prefrontal cortex in the foreperiod effect: TMs evidence for dual mechanisms in temporal preparation. Cerebral Cortex, 17(2), 466-474.

Van Ede, F., M. Niklaus, \& A.C. Nobre (2017). Temporal expectations guide dynamic prioritization in visual working memory through attenuated $\alpha$ oscillations. Journal of Neuroscience, 37(2), 437-445.

Woodrow, H. (1914). The measurement of attention. Psychological Monographs, 17, 158. Zanto, T.P., P. Pan, H. Liu, J. Bollinger, A.C. Nobre, \& A. Gazzaley (2011). Age-related changes in orienting attention in time. Journal of Neuroscience, $37(35), 12461-12470$. 